\title{
Slip Length Dependent Propulsion Speed of Catalytic Colloidal Swimmers near Walls
}

\author{
Stefania Ketzetzi, ${ }^{1}$ Joost de Graaf, ${ }^{2}$ Rachel P. Doherty, ${ }^{1}$ and Daniela J. Kraft ${ }^{1}$ \\ ${ }^{1}$ Soft Matter Physics, Huygens-Kamerlingh Onnes Laboratory, Leiden University, \\ P.O. Box 9504, 2300 RA Leiden, The Netherlands \\ ${ }^{2}$ Institute for Theoretical Physics, Center for Extreme Matter and Emergent Phenomena, \\ Utrecht University, Princetonplein 5, 3584 CC Utrecht, The Netherlands
}

(Received 20 December 2018; revised manuscript received 6 September 2019; published 28 January 2020)

\begin{abstract}
Catalytic colloidal swimmers that propel due to self-generated fluid flows exhibit strong affinity for surfaces. Here, we report experimental measurements of a significant dependence of such microswimmers' speed on the nearby substrate material. We find that speeds scale with the solution contact angle $\theta$ on the substrate, which relates to the associated hydrodynamic substrate slip length, as $V \propto(\cos \theta+1)^{-3 / 2}$. We show that such dependence can be attributed to osmotic coupling between swimmers and substrate. Our work points out that hydrodynamic slip at nearby walls, though often unconsidered, can significantly impact microswimmer self-propulsion.
\end{abstract}

DOI: 10.1103/PhysRevLett.124.048002

Colloidal swimmers constitute a new class of nonequilibrium model systems that also hold great promise for applications owing to their fast directed motion in liquid environments. A simple experimental realization of such microswimmers are spherical colloids half coated with $\mathrm{Pt}$ [1]. These colloids move autonomously in $\mathrm{H}_{2} \mathrm{O}_{2}$ solutions due to asymmetric catalytic reactions taking place on their surfaces [2] and are typically found self-propelling parallel to a substrate [3-6]. This substrate affinity leads to accumulation [3] and retention [5-7] of swimmers at surfaces, such as walls and obstacles, and can be exploited as a means to guide their motion $[5,8]$.

Strikingly, upon approaching a surface, numerical and theoretical models predict both an increase or decrease in swimming speed depending on the considered propulsion mechanism and the physicochemical properties of the swimmer and wall [9-15]. At the same time, experimental observations also hint at non-negligible substrate effects on the speed of synthetic swimmers [16-18]. In fact, substrate effects may be at the heart of inconsistencies in catalytic microswimmer speeds under comparable experimental conditions. For example, speeds as disparate as $3 \mu \mathrm{m} / \mathrm{s}$ [19] and $18 \mu \mathrm{m} / \mathrm{s}$ [3] were found for polystyrene spheres with $5 \mathrm{~nm} \mathrm{Pt}$ coating in $10 \% \mathrm{H}_{2} \mathrm{O}_{2}$. This difference is even more surprising when one considers that the slower speeds were observed for the smaller species, whereas the speed of Pt-coated swimmers should scale inversely with size [20].

Published by the American Physical Society under the terms of the Creative Commons Attribution 4.0 International license. Further distribution of this work must maintain attribution to the author(s) and the published article's title, journal citation, and DOI.
Recent measurements on different polymer-coated substrates revealed a propulsion speed decrease upon functionalization with either positively or negatively charged polyelectrolytes for bimetallic swimmers [16]. This is puzzling because contrary to most current predictions it indicates that the wall zeta potential does not have a dominant effect on the speed of self-electrophoretic swimmers. Furthermore, photoactivated $\mathrm{TiO}_{2} / \mathrm{SiO}_{2}$ swimmers were found to swim with $3 \mu \mathrm{m} / \mathrm{s}$ speed on glass, while they propelled with $4 \mu \mathrm{m} / \mathrm{s}$ speed on gold (Au) coated glass substrates [18]. It was proposed, based on zeta potential values for $\mathrm{Au}$ and glass at neutral $p \mathrm{H}$ conditions, that the increase in the propulsion speed stemmed from the lower zeta potential of the Au surface. However, neutral conditions are likely not met in $\mathrm{H}_{2} \mathrm{O}_{2}$ solutions. Results obtained using Au-coated substrates are hard to interpret, because $\mathrm{Au}$ could in principle catalyze $\mathrm{H}_{2} \mathrm{O}_{2}$ decomposition and therefore interfere with the propulsion reaction [18]. To elucidate the origin of these intriguing observations, other surfaces ought to be examined in a quantitative manner. Understanding potential surface effects on colloid self-propulsion is essential not only for their use as model systems and the development of a quantitative framework but also for future applications that may require motion in complex environments comprising obstacles or confining walls [21].

In this Letter, we quantitatively examine the effect of various substrates, namely glass, glass coated with the organosilicon compound polydimethylsiloxane (PDMS), and plastic substrates made of a polyethylene (PE) or polystyrene (PS) derivative, on the speed of catalytic colloidal swimmers. Under otherwise fixed conditions, we observe significant differences in propulsion speeds, which cannot be fully accounted for by the substrate zeta 


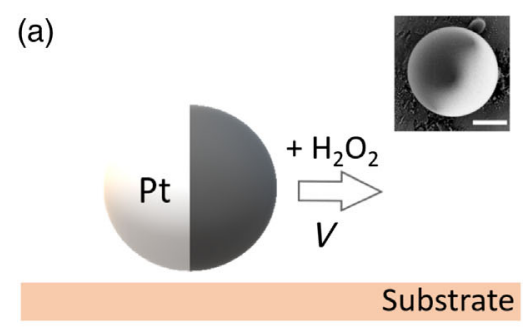

(b)

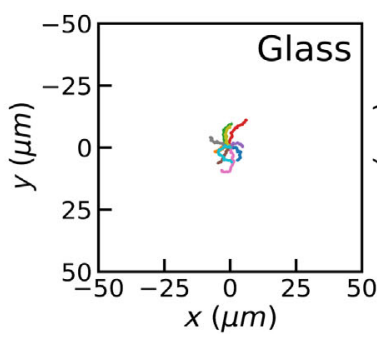

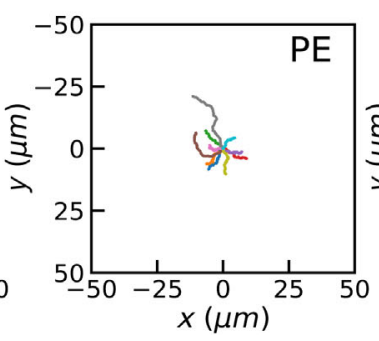

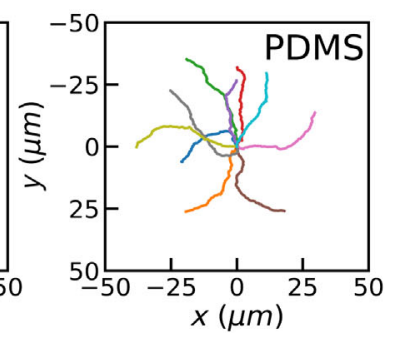

FIG. 1. Influence of the substrate on colloid self-propulsion. (a) Schematic of the experimental setup. The self-propulsion of $2.7 \mu \mathrm{m}$ diameter Pt-coated colloids was observed in the same $\mathrm{H}_{2} \mathrm{O}_{2}$ solution and under fixed conditions on various substrates. All colloids were taken from the same batch. A scanning electron microscope image of a representative colloid is shown in the inset, with the brighter hemisphere indicating the Pt coating. Scale bar is $1 \mu \mathrm{m}$. (b) Typical $8 \mathrm{~s}$ active colloid trajectories on glass, polyethylene (PE) and polydimethylsiloxane (PDMS).

potential. Instead, we find that speeds upon different substrates fall on a single curve as a function of the solution-substrate contact angle which relates to the substrate slip length [22]. After careful examination of the observed dependence in view of qualitative and scaling arguments, and accounting for possible couplings between swimmers and the substrate, we show that substratedependent speeds may result from osmotic coupling.

For all experiments, we used TPM colloids [23] of $2.7 \mu \mathrm{m}$ diameter half-coated with $4.9 \mathrm{~nm}$ of Pt by sputtercoating, see inset in Fig. 1(a). Colloids were prepared in one batch, and thus any inhomogeneities arising from their preparation, including $\mathrm{Pt}$ thickness that affects $\mathrm{H}_{2} \mathrm{O}_{2}$ decomposition, should be universal. Measurements were taken with a 60x ELWD air objective (NA 0.7) on an inverted Nikon TI-E microscope at $18.92 \mathrm{fps}$ in the dark typically within the hour after dispersing the colloids at dilute particle concentration $\left(\approx 10^{-7} \mathrm{w} / \mathrm{v}\right)$ in deionized water containing $10 \% \mathrm{H}_{2} \mathrm{O}_{2}$. The colloids quickly reached the lower surface and continued to self-propel parallel to it, as illustrated in Fig. 1(a). Figure 1(b) shows 10 representative $x y$ trajectories, obtained following [24], on glass, PE, and PDMS substrates acquired over a time interval of $8 \mathrm{~s}$. We find that the colloids cover significantly greater distances on PDMS than on glass and PE, clearly demonstrating that the substrate affects colloid motion.

To quantify the differences in the observed behavior, we first obtain the speed $V$ of each individual colloid from its short-term mean squared displacement following Ref. [19]. We fit the corresponding probability density function (PDF) of the speed with a log-normal distribution following Ref. [25] to obtain the speed distribution parameters on each substrate. The most frequently encountered speeds, as obtained from the fitted peak position of each PDF, are $1.05 \pm 0.09,1 \pm 0.2$, and $2.8 \pm 0.3 \mu \mathrm{m} / \mathrm{s}$, above glass, $\mathrm{PE}$, and PDMS, respectively. Interestingly, though all three substrates are chemically different, the colloids show similar speeds for two of the substrates and a notably different speed for the third. At the same time, the characteristic time scale for rotation $\tau_{R}[19,26,27]$ is similar for all three substrates. Details on the determination of $V$ and $\tau_{R}$ can be found in the Supplemental Material [28], Sec. I D. In the absence of $\mathrm{H}_{2} \mathrm{O}_{2}$, however, the translational diffusion coefficients are similar, namely $0.099 \pm 0.005$, $0.098 \pm 0.008$, and $0.105 \pm 0.005 \mu \mathrm{m}^{2} / \mathrm{s}$, for glass, PE and PDMS, respectively. Thus, substrate-dependent differences arise only in the active state.

While speeds may be influenced by colloid properties, such as size [20], roughness [29], and slip [30,31], these effects are negligible here since the same colloid batch was used in all experiments. Therefore, the observed speed differences arise from differences in the substrate properties. To quantitatively unravel the origin of our observations, we consider substrate properties that may influence colloid motion. The fluid flow generated by the anisotropic catalytic reaction on the swimmer surface [32], and hence the swimmer's propulsion speed [33], has been predicted to be affected by the swimmer-wall distance [9-15], wall zeta potential [11,18] and wall surface inhomogeneities [18]. Surprisingly, little consideration has been given until now on whether slip on the substrate impacts propulsion speeds, even though slip on the colloid has already been shown to do so [31]. Considering that hydrodynamic attraction in the active state pulls the colloids close to surfaces, to the extent that they even propel along the top of their container [3], colloid-substrate distances are expected to be small. Ptcoated swimmers of $2.5 \mu \mathrm{m}$ radius have been found to not swim over $200 \mathrm{~nm}$ steps [6], and other experiments pointed out that distances may even be of the order of tens of $\mathrm{nm}$ $[34,35]$. Since wall slip lengths ranging from several [36$39]$ to hundreds $[40,41]$ of nanometers and even micrometers [42] have been reported, boundary conditions could strongly affect the speed. Following Ref. [30], we hypothesize that deviations from the no-slip condition on the substrate may enhance nearby swimmers' speeds.

Surface slip relates to liquid-solid interactions and thus surface wetting properties, and generally, though not always, increases with increasing hydrophobicity and thus contact angle $\theta[22,38,39,43]$. Since hydrophobic surfaces possess a larger slip fluid velocity [44], we hypothesize that they also lead to a higher propulsion speed. Conversely, the no-slip approximation on hydrophilic surfaces would lead 
(a)

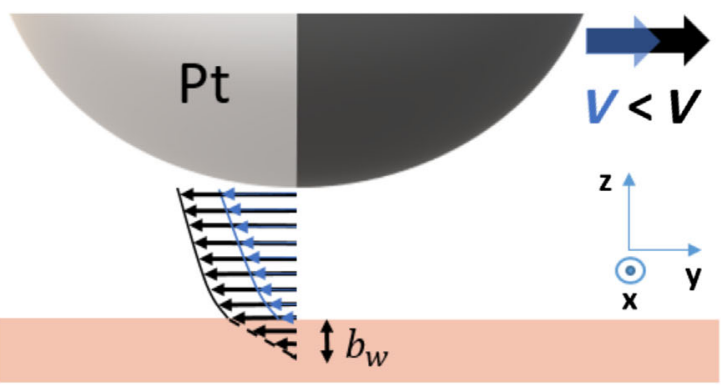

(b)

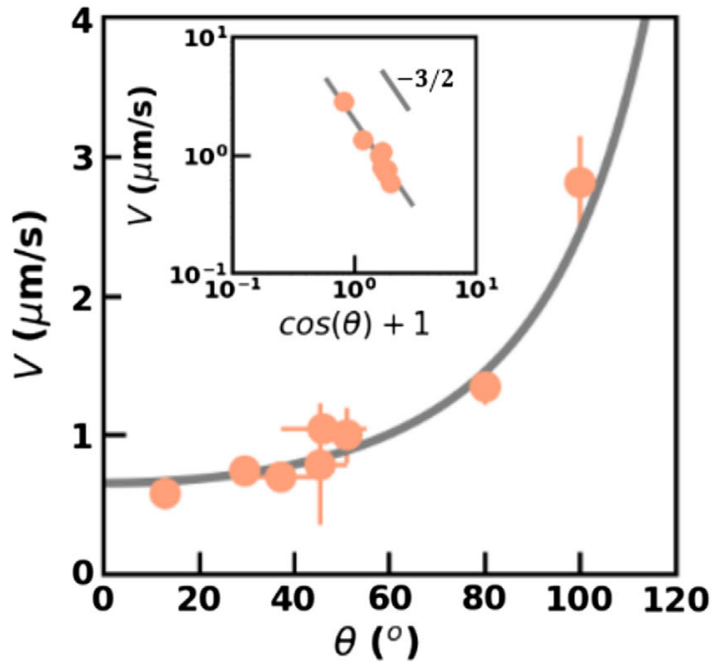

FIG. 2. Propulsion speed $V$ of catalytic microswimmers on surfaces with different contact angle $\theta$. (a) Schematic of the proposed model. At a given distance from the substrate, the propulsion speed $V$ resulting from the colloid-generated fluid flow is larger on a hydrophobic substrate due to the larger slip length $b_{w}$ (not to scale). Here, only the fluid flow velocity profile due to hydrodynamic slip on the wall is illustrated. (b) $V$ as a function of $\theta$ and least squares fit (solid line) with $V=A(\cos \theta+1)^{-3 / 2}$ that follows from our model, with $A=1.84 \mu \mathrm{m} / \mathrm{s}$. The inset shows the data on a log-log scale.

to lower speeds, see Fig. 2(a). Indeed, the advancing contact angle measured for the $\mathrm{H}_{2} \mathrm{O}_{2}$ solution by the sessile drop method agrees with this hypothesis: $\theta$ is $46 \pm 9^{\circ}$, $51 \pm 3^{\circ}$, and $100 \pm 3^{\circ}$, for glass, PE, and PDMS (as in [45]), respectively. PE is normally hydrophobic; thus a modification has been performed by the supplier.

To further test this hypothesis, we modulated the hydrophilicity of the employed substrates and repeated the experiments. We increased the hydrophilicity of glass by either a cleaning procedure $\left(\theta=29.5 \pm 3^{\circ}\right)$ or treatment with $\mathrm{HCl}$ [46] $\left(\theta=13 \pm 3^{\circ}\right)$ and we observed a concomitant speed decrease by $30 \%$ and $45 \% \quad(V=0.78 \pm 0.03$ and $V=0.6 \pm 0.05 \mu \mathrm{m} / \mathrm{s}$ ), respectively. Conversely, when we rendered the glass more hydrophobic $\left(\theta=80 \pm 2^{\circ}\right)$, we found that speed increased by $28 \%(V=1.35 \pm 0.13 \mu \mathrm{m} / \mathrm{s})$ compared to untreated glass. Similar behavior was seen on PDMS that was rendered hydrophilic through UV-ozone treatment [47,48] $\left(\theta=37 \pm 7^{\circ}\right)$ : colloids propelled four times slower $(V=0.7 \pm 0.05 \mu \mathrm{m} / \mathrm{s})$ than on hydrophobic PDMS. Finally, we employed commercially available hydrophilic PS substrates $\left(\theta=46 \pm 6^{\circ}\right)$ and found $V=0.8 \pm 0.45 \mu \mathrm{m} / \mathrm{s}$. We summarize these findings by plotting $V$ as a function of $\theta$ in Fig. 2(b). The collapse of the data onto a single curve suggests that $\theta$, is the most relevant parameter and that other differences among substrates, besides their effect on $\theta$, are of lesser importance.

Next, we develop a quantitative framework for the slipdependent propulsion speeds. For our analysis we consider that the height above the substrate remains relatively unaffected by the change of substrate, as supported by our experimental measurements of the diffusion coefficient [49-51], see the Supplemental Material [28], Sec. ID. When the height is left unperturbed by varying $\cos \theta-$ possibly due to electrostatic or even hydrodynamic coupling - the dominant source of change to the propulsion speed comes from solute gradients near the substrate. As mentioned earlier, these are generated by reactions taking place on the swimmer surface and, similar to the way they cause self-propulsion, lead to an effective surface fluid velocity along the wall [33]. This is often referred to as "slip" velocity, but we do not use this term to avoid confusion with the concept of hydrodynamic surface slip. This effective surface fluid velocity couples back to the swimmer, modifying its net velocity $[5,8,11,52]$. In the Supplemental Material [28], Sec. II, we show that neither purely hydrodynamic coupling $[15,53,54]$, solute confinement $[9,10,12-14]$, nor reaction-based coupling [20] can account for the significant wall effect. Instead, as we will show, our observation can be attributed to osmotic coupling $[5,8,11,33,52,55]$.

The osmotic coupling scales linearly with the slipvelocity parameter $\xi_{w}$, i.e., the prefactor that converts solute gradients into effective hydrodynamic surface velocities [33]. Ajdari and Bocquet [30] have shown that for a partial-slip wall the result by Anderson [33] can be generalized to

$$
\xi_{w}=\left(k_{B} T / \mu\right) \lambda_{w} \gamma_{w}\left(1+b_{w} / \lambda_{w}\right),
$$

where slippage is expressed by the slip length $b_{w} ; b_{w}=0$ for a no-slip surface and $b_{w} \rightarrow \infty$ for a full-slip surface. Here, we have introduced $k_{B}$ Boltzmann's constant, $T$ the temperature, $\mu$ the dynamic viscosity, $\lambda_{w}$ a length scale for the solute-surface interactions, and $\gamma_{w}$ a length measuring the solute excess [30]. For smooth surfaces, as we consider here, the value $\lambda_{w}$ is left relatively unaffected by changes in $\theta$, but $b_{w} \propto(1+\cos \theta)^{-2}$ and $\gamma_{w} \propto \sqrt{1+\cos \theta}$ [22]. This leads to the following leading-order proportionality of the measured speed with $\theta$ :

$$
V \propto(1+\cos \theta)^{-3 / 2},
$$

which requires that $b_{w} / \lambda_{w} \gg 1$, see also the Supplemental Material [28], Sec. II, for a more in-depth discussion on the 


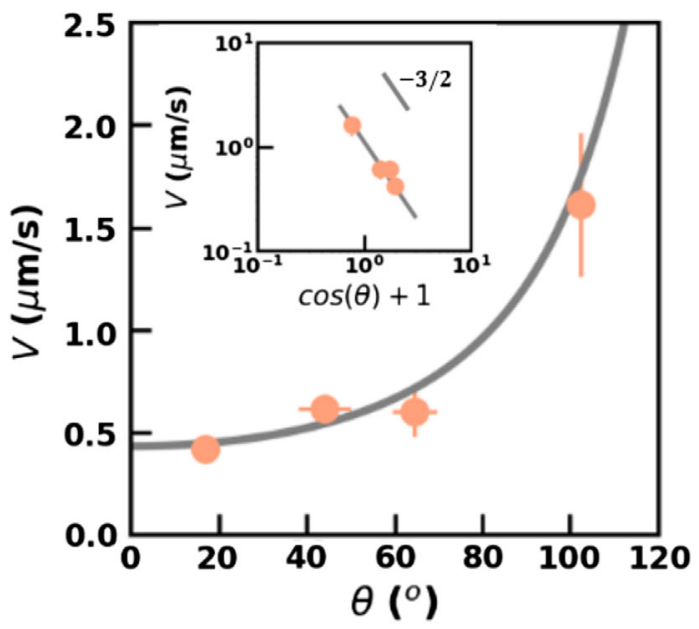

FIG. 3. Slip dependence of the propulsion speed of colloidal swimmers in salt solution. Average speed in $1 \mathrm{mM} \mathrm{NaCl}$ as a function of the contact angle $\theta$ and least squares fit (solid line) with $V=A(\cos \theta+1)^{-3 / 2}$ that follows from our model, with $A$ $1.2 \mu \mathrm{m} / \mathrm{s}$. The inset shows the data on a log-log scale.

osmotic coupling based mechanism, which additionally includes Refs. [56-60]. We use this quantitative relationship between propulsion speed and contact angle to fit the experimental data presented in Fig. 2(b). The proportionality factor $A$, which contains all other contributions to the speed that are slip independent, is $1.84 \mu \mathrm{m} / \mathrm{s}$. The excellent agreement between data and model further quantitatively corroborates the influence of slip.

To provide additional support to our hypothesis, we test whether the above dependence persists in the presence of salt. Previous experiments employing $2 \mu \mathrm{m}$ PS spheres showed that even $1 \mathrm{mM}$ salt considerably decreases propulsion speeds $[3,61]$. Although speeds for similar $\mathrm{H}_{2} \mathrm{O}_{2}$ concentration without salt were different above glass, namely around 4 [61] and $18 \mu \mathrm{m} / \mathrm{s}$ [3], they reduced to 0.45 and $1 \mu \mathrm{m} / \mathrm{s}$, respectively, in $1 \mathrm{mM}$ salt. In agreement with these experiments, we find that speeds above different substrates decrease with added salt, see Fig. 3. More importantly, we observe that speeds still follow the same slip dependence. In salt solution, the proportionality factor $A$ is $1.2 \mu \mathrm{m} / \mathrm{s}$; i.e., it shows a $33 \%$ decrease compared with the salt-free case. Considering that the influence of salt is complex, potentially affecting zeta potentials, separation, higher-order hydrodynamic moments or possibly more properties including bulk speeds, this decrease is not surprising. However, that the same dependence persists strongly supports the importance of slip and may provide additional insights into the propulsion mechanism [3].

We emphasize that other substrate properties besides slip may affect propulsion speeds. As mentioned earlier, lowering the substrate zeta potential has been proposed to increase speeds [18]. For completeness we thus measured substrate zeta potentials using a surface zeta potential cell from Malvern by laser Doppler electrophoresis following Ref. [62] using tracers prepared as in [63], see the Supplemental Material [28], Sec. IE. We find zeta potentials of $-38.3 \pm 1.1$ and $-22 \pm 0.9 \mathrm{mV}$ for glass and PDMS, respectively, in line with this proposal. However, we find an even lower zeta potential, $-11 \pm 5 \mathrm{mV}$, for hydrophilic PDMS. Due to the low speed on hydrophilic PDMS, we conclude that the substrate zeta potential is, surprisingly, not the dominant effect. Second, an increase in the substrate roughness was shown to increase the speed [18]. We thus performed atomic force microscopy (AFM) measurements, see the Supplemental Material [28], Sec. I F. The average substrate roughness $\mathrm{Ra}$ is 1.5 and $5 \mathrm{~nm}$, for glass and PDMS, respectively, with Ra denoting the arithmetic mean of the deviations in height from the roughness mean value. However, hydrophilic PDMS, with a roughness equal or higher [64] to untreated PDMS, featured lower speeds. We thus conclude that substrate roughness is also not the dominant effect here.

Our experiments also provide a new perspective on previous work: the speed increase previously observed on Au-coated surfaces [18] may be due to increased surface slip, since contact angles on Au are typically higher than on glass $[65,66]$. Similarly, the speed decrease in [16] may be due to the hydrophilic polyelectrolyte coatings employed on the glass. Besides, our findings may explain the discrepancies in reported speeds between previous experiments. Even though glass substrates were used in all cases, glass can differ in composition, homogeneity, and hydrophilicity due to different preparation, coatings, treatment, and cleaning methods from the supplier or the researchers themselves, as we have also demonstrated above. We also found that contact angles sometimes varied by $10^{\circ}$ within the same type of and/or different parts of the same substrate. AFM indicated that this is likely due to inhomogeneous application or even local absence of coatings applied by the supplier. If the coating or substrate treatment is inhomogeneous or unstable, for example due to a chemical reaction with $\mathrm{H}_{2} \mathrm{O}_{2}$, a locally or temporally different substrate slip can be observed. For example, we found a $15^{\circ}$ increase in contact angle for the PS substrate before and after being exposed to $\mathrm{H}_{2} \mathrm{O}_{2}$ for several hours.

In conclusion, we show that the propulsion speed of catalytic microswimmers near a wall is influenced by the wall slip boundary condition. This quantitatively follows from theoretical predictions on the basis of an osmotic coupling mechanism, indicating further control and understanding of the behavior of self-propelled particles. In future work, it would be interesting to investigate if slip affects other features of active motion as well, such as the orientation of active particles with respect to the wall [67]. The here discussed slip dependence of the propulsion speed should not only be relevant for catalytic swimmers but any microswimmer that creates a fluid flow in the vicinity of a substrate. 
We thank Malvern for providing the Surface Zeta Potential Cell and Sandra Remijn for help with the substrate zeta potential measurements. We thank Federica Galli for useful discussions and help with the AFM measurements, and Nikos Oikonomeas and Ruben Verweij for discussion and help with python routines. We are grateful to Aidan Brown, Willem Boon, and Jeroen Rodenburg for fruitful discussions. J. d. G. thanks NWO for funding through StartUp Grant No. 740.018.013 and through association with the EU-FET Project No. NANOPHLOW (766972) within Horizon 2020. D. J. K. gratefully acknowledges funding from the European Research Council (ERC) under the European Union's Horizon 2020 research and innovation program (Grant Agreement No. 758383).

[1] S. J. Ebbens and D. A. Gregory, Catalytic janus colloids: Controlling trajectories of chemical microswimmers, Acc. Chem. Res. 51, 1931 (2018).

[2] R. Golestanian, T. B. Liverpool, and A. Ajdari, Propulsion of a Molecular Machine by Asymmetric Distribution of Reaction Products, Phys. Rev. Lett. 94, 220801 (2005).

[3] A. Brown and W. Poon, Ionic effects in self-propelled Ptcoated janus swimmers, Soft Matter 10, 4016 (2014).

[4] H. Ke, S. Ye, R. L. Carroll, and K. Showalter, Motion analysis of self-propelled Pt-silica particles in hydrogen peroxide solutions, J. Phys. Chem. A 114, 5462 (2010).

[5] S. Das, A. Garg, A. I. Campbell, J. Howse, A. Sen, D. Velegol, R. Golestanian, and S. J. Ebbens, Boundaries can steer active janus spheres, Nat. Commun. 6, 8999 (2015).

[6] J. Simmchen, J. Katuri, W. E. Uspal, M. N. Popescu, M. Tasinkevych, and S. Sánchez, Topographical pathways guide chemical microswimmers, Nat. Commun. 7, 10598 (2016).

[7] A. T. Brown, I. D. Vladescu, A. Dawson, T. Vissers, J. Schwarz-Linek, J. S. Lintuvuori, and W. C. K. Poon, Swimming in a crystal, Soft Matter 12, 131 (2016).

[8] W. E. Uspal, M. N. Popescu, S. Dietrich, and M. Tasinkevych, Guiding Catalytically Active Particles with Chemically Patterned Surfaces, Phys. Rev. Lett. 117, 048002 (2016)

[9] M. N. Popescu, S. Dietrich, and G. Oshanin, Confinement effects on diffusiophoretic self-propellers, J. Chem. Phys. 130, 194702 (2009).

[10] D. G. Crowdy, Wall effects on self-diffusiophoretic janus particles: A theoretical study, J. Fluid Mech. 735, 473 (2013).

[11] T.-Y. Chiang and D. Velegol, Localized electroosmosis (LEO) induced by spherical colloidal motors, Langmuir 30, 2600 (2014).

[12] W. E. Uspal, M. N. Popescu, S. Dietrich, and M. Tasinkevych, Self-propulsion of a catalytically active particle near a planar wall: From reflection to sliding and hovering, Soft Matter 11, 434 (2015).

[13] Y. Ibrahim and T. B. Liverpool, The dynamics of a selfphoretic janus swimmer near a wall, Eur. Phys. Lett. 111, 48008 (2015).

[14] A. Mozaffari, N. Sharifi-Mood, J. Koplik, and C. Maldarelli, Self-diffusiophoretic colloidal propulsion near a solid boundary, Phys. Fluids 28, 053107 (2016).
[15] Z. Shen, A. Würger, and J. S. Lintuvuori, Hydrodynamic interaction of a self-propelling particle with a wall. Comparison between an active janus particle and a squirmer model, Eur. Phys. J. E 41, 39 (2018).

[16] M. Wei, C. Zhou, J. Tang, and W. Wang, Catalytic micromotors moving near polyelectrolyte-modified substrates: The roles of surface charges, morphology, and released ions, ACS Appl. Mater Interfaces 10, 2249 (2018).

[17] J. Palacci, S. Sacanna, S.-H. Kim, G.-R. Yi, D. J. Pine, and P. M. Chaikin, Light-activated self-propelled colloids, Phil. Trans. R. Soc. A 372, 2249 (2014).

[18] A. L. Holterhoff, M. Li, and J. G. Gibbs, Self-phoretic microswimmers propel at speeds dependent upon an adjacent surface's physicochemical properties, J. Phys. Chem. Lett. 9, 5023 (2018).

[19] J. R. Howse, R. A. L. Jones, A. J. Ryan, T. Gough, R. Vafabakhsh, and R. Golestanian. Self-Motile Colloidal Particles: From Directed Propulsion to Random Walk, Phys. Rev. Lett. 99, 048102 (2007).

[20] S. Ebbens, M.-H. Tu, J. R. Howse, and R. Golestanian, Size dependence of the propulsion velocity for catalytic janussphere swimmers, Phys. Rev. E 85, 020401 (2012).

[21] C. Bechinger, R. Di Leonardo, H. Löwen, C. Reichhardt, G. Volpe, and G. Volpe, Active particles in complex and crowded environments, Rev. Mod. Phys. 88, 045006 (2016).

[22] D. M. Huang, C. Sendner, D. Horinek, R. R. Netz, and L. Bocquet, Water Slippage Versus Contact Angle: A Quasiuniversal Relationship, Phys. Rev. Lett. 101, 226101 (2008).

[23] C. van der Wel, R. K. Bhan, R. W. Verweij, H. C. Frijters, Z. Gong, A. D. Hollingsworth, S. Sacanna, and D. J. Kraft, Preparation of colloidal organosilica spheres through spontaneous emulsification, Langmuir 33, 8174 (2017).

[24] D. B. Allan, T. Caswell, N. C. Keim, and C. M. van der Wel, trackpy: Trackpy v0.4.1 (2018), https://www.doi.org/10 .5281 /zenodo. 1226458 .

[25] M. Lisicki, M. F. V. Rodrigues, R. E. Goldstein, and E. Lauga, Swimming eukaryotic microorganisms exhibit a universal speed distribution, eLife 8, e44907 (2019).

[26] S. Ebbens, R. A. L. Jones, A. J. Ryan, R. Golestanian, and J. R. Howse, Self-assembled autonomous runners and tumblers, Phys. Rev. E 82, 015304(R) (2010).

[27] X. Wang, M. In, C. Blanc, M. Nobili, and A. Stocco, Enhanced active motion of janus colloids at the water surface, Soft Matter 11, 7376 (2015).

[28] See the Supplemental Material at http://link.aps.org/ supplemental/10.1103/PhysRevLett.124.048002 for experimental details on sample preparation and analysis, surface zeta potential, atomic force and contact angle measurements, as well as theoretical details on osmotic coupling induced speed variation.

[29] B. W. Longbottom and S. A. F. Bon, Improving the engine power of a catalytic janus-sphere micromotor by roughening its surface, Sci. Rep. 8, 4622 (2018).

[30] A. Ajdari and L. Bocquet, Giant Amplification of Interfacially Driven Transport by Hydrodynamic Slip: DiffusioOsmosis and Beyond, Phys. Rev. Lett. 96, 186102 (2006).

[31] M. Manjare, Y. T. Wu, B. Yang, and Y.-P. Zhao, Hydrophobic catalytic janus motors: Slip boundary condition and enhanced catalytic reaction rate, Appl. Phys. Lett. 104, 054102 (2014). 
[32] A. I. Campbell, S. J. Ebbens, P. Illien, and R. Golestanian, Experimental observation of flow fields around active janus spheres, Nat. Commun. 10, 3952 (2019).

[33] J. L. Anderson, Colloid transport by interfacial forces, Annu. Rev. Fluid Mech. 21, 61 (1989).

[34] D. Takagi, A. B. Braunschweig, J. Zhang, and M. J. Shelley, Dispersion of Self-Propelled Rods Undergoing FluctuationDriven Flips, Phys. Rev. Lett. 110, 038301 (2013).

[35] D. Takagi, J. Palacci, A. B. Braunschweig, M. J. Shelley, and J. Zhang, Hydrodynamic capture of microswimmers into sphere-bound orbits, Soft Matter 10, 1784 (2014).

[36] V. S. J. Craig, C. Neto, and D. R. M. Williams, ShearDependent Boundary Slip in an Aqueous Newtonian Liquid, Phys. Rev. Lett. 87, 054504 (2001).

[37] J. Baudry, E. Charlaix, A. Tonck, and D. Mazuyer, Experimental evidence for a large slip effect at a nonwetting fluid-solid interface, Langmuir 17, 5232 (2001).

[38] B. Bhushan, Y. Wang, and A. Maali, Boundary slip study on hydrophilic, hydrophobic, and superhydrophobic surfaces with dynamic atomic force microscopy, Langmuir 25, 8117 (2009).

[39] L. Bocquet and J.-L. Barrat, Flow boundary conditions from nano- to micro-scales, Soft Matter 3, 685 (2007).

[40] R. Pit, H. Hervet, and L. Léger, Direct Experimental Evidence of Slip in Hexadecane: Solid Interfaces, Phys. Rev. Lett. 85, 980 (2000).

[41] C. Cottin-Bizonne, S. Jurine, J. Baudry, J. Crassous, F. Restagno, and E. Charlaix, Nanorheology: An investigation of the boundary condition at hydrophobic and hydrophilic interfaces, Eur. Phys. J. E 9, 47 (2002).

[42] Y. Zhu and S. Granick, Rate-Dependent Slip of Newtonian Liquid at Smooth Surfaces, Phys. Rev. Lett. 87, 096105 (2001).

[43] B. Siboulet, J. Molina, B. Coasne, P. Turq, and J.-F. Dufreche, Water self-diffusion at the surface of silica glasses: Effect of hydrophilic to hydrophobic transition, Mol. Phys. 111, 3410 (2013).

[44] M. T. Kezirian, Hydrodynamics with a wall-slip boundary condition for a particle moving near a plane wall bounding a semi-infinite viscous fluid, $\mathrm{Ph}$. D. thesis, Massachusetts Institute of Technology, 1992.

[45] V. Jokinen, P. Suvanto, and S. Franssila, Oxygen and nitrogen plasma hydrophilization and hydrophobic recovery of polymers, Biomicrofluidics 6, 016501 (2012).

[46] J. J. Cras, C. A. Rowe-Taitt, D. A. Nivens, and F. S. Ligler, Comparison of chemical cleaning methods of glass in preparation for silanization, Biosens. Bioelectron. 14, 683 (1999).

[47] K. Efimenko, W. E. Wallace, and J. Genzer, Surface modification of sylgard-184 poly(dimethyl siloxane) networks by ultraviolet and ultraviolet/ozone treatment, J. Colloid Interface Sci. 254, 306 (2002).

[48] A. Oláh, H. Hillborg, and G. J. Vancso, Hydrophobic recovery of UV/ozone treated poly(dimethylsiloxane): Adhesion studies by contact mechanics and mechanism of surface modification, Appl. Surf. Sci. 239, 410 (2005).

[49] A. J. Goldman, R. G. Cox, and H. Brenner, Slow viscous motion of a sphere parallel to a plane wall-I Motion through a quiescent fluid, Chem. Eng. Sci. 22, 637 (1967).
[50] P. Sharma, S. Ghosh, and S. Bhattacharya, A high-precision study of hindered diffusion near a wall, Appl. Phys. Lett. 97, 104101 (2010).

[51] C. Ha, H. D. Ou-Yang, and H. Kyu Pak, Direct measurements of colloidal hydrodynamics near flat boundaries using oscillating optical tweezers, Physica (Amsterdam) 392A, 3497 (2013).

[52] M. N. Popescu, W. E. Uspal, A. Domínguez, and S. Dietrich, Effective interactions between chemically active colloids and interfaces, Acc. Chem. Res. 51, 2991 (2018).

[53] S. E. Spagnolie and E. Lauga, Hydrodynamics of selfpropulsion near a boundary: Predictions and accuracy of far-field approximations, J. Fluid Mech. 700, 105 (2012).

[54] J. S. Lintuvuori, A. T. Brown, K. Stratford, and D. Marenduzzo, Hydrodynamic oscillations and variable swimming speed in squirmers close to repulsive walls, Soft Matter 12, 7959 (2016).

[55] H. J. Keh and S. B. Chen, Electrophoresis of a colloidal sphere parallel to a dielectric plane, J. Fluid Mech. 194, 377 (1988).

[56] A. J. T. M. Mathijssen, A. Doostmohammadi, J. M. Yeomans, and T. N. Shendruk, Hydrodynamics of micro-swimmers in films, J. Fluid Mech. 806, 35 (2016).

[57] E. Lauga and T. M. Squires, Brownian motion near a partialslip boundary: A local probe of the no-slip condition, Phys. Fluids 17, 103102 (2005).

[58] H. Loussaief, L. Pasol, and F. Feuillebois, Motion of a spherical particle in a viscous fluid along a slip wall, Q. J. Mech. Appl. Math. 68, 115 (2015).

[59] M. J. Lighthill, On the squirming motion of nearly spherical deformable bodies through liquids at very small Reynolds numbers, Commun. Pure Appl. Math. 5, 109 (1952).

[60] J. R. Blake, A spherical envelope approach to ciliary propulsion, J. Fluid Mech. 46, 199 (1971).

[61] S. Ebbens, D. A. Gregory, G. Dunderdale, J. R. Howse, Y. Ibrahim, T. B. Liverpool, and R. Golestanian, Electrokinetic effects in catalytic platinum-insulator janus swimmers, Europhys. Lett. 106, 58003 (2014).

[62] J. C. W. Corbett, F. McNeil-Watson, R. O. Jack, and M. Howarth, Measuring surface zeta potential using phase analysis light scattering in a simple dip cell arrangement, Colloids Surf. A 396, 169 (2012).

[63] C. van der Wel, N. Bossert, Q. J. Mank, M. G. T. Winter, D. Heinrich, and D. J. Kraft, Surfactant-free colloidal particles with specific binding affinity, Langmuir 33, 9803 (2017).

[64] R. Raveendran and M. A. G. Namboothiry, Surface-treated poly(dimethylsiloxane) as a gate dielectric in solutionprocessed organic field-effect transistors, ACS Omega 3, 11278 (2018).

[65] R. A. Erb, The wettability of gold, J. Phys. Chem. 72, 2412 (1968).

[66] M. E. Abdelsalam, P. N. Bartlett, T. Kelf, and J. Baumberg, Wetting of regularly structured gold surfaces, Langmuir 21, 1753 (2005).

[67] K. Dietrich, D. Renggli, M. Zanini, G. Volpe, I. Buttinoni, and L. Isa, Two-dimensional nature of the active brownian motion of catalytic microswimmers at solid and liquid interfaces, New J. Phys. 19, 065008 (2017). 